\title{
Saphenous Vein
}

National Cancer Institute

\section{Source}

National Cancer Institute. Saphenous Vein. NCI Thesaurus. Code C33511.

One of two (Greater, Small) superficial veins of the leg and thigh. 